\title{
Evaluation of the pattern of self-medication among the paramedical support staff in a tertiary care hospital
}

\author{
Gala Khushboo S1, Sinha Shyamal R', Mankeshwar Ranjit', Desai Viral K' and Gala Ritu S² \\ 'Department of Pharmacology, Grant Medical College and Sir J. J. Group of hospitals, Mumbai, INDIA. \\ 2PACE College, Mumbai, INDIA.
}

\begin{abstract}
Objective: To evaluate self-medication practices in the support staff of a tertiary care hospital. Methods: It was a cross-sectional study in which a self-developed questionnaire was used. Study population consisted of two categories of paramedical staff (Category l: Nurses, Category II: Non nursing staff - technicians, clerks). Results: It was found that $68 \%$ of Cat I and $52 \%$ of Cat II subjects practised self-medication. Self-medication with analgesics was the most prevalent. A high percentage of the nursing staff was found to be self-medicating with antibiotics although the awareness levels about antibiotic resistance are high among them. Nearly $50 \%$ of the subjects selfmedicated with nutritional supplements and there was a greater tendency to select the nutritional supplement by means of advertisements. Most subjects in both categories stopped taking their medication immediately after cessation of symptoms, reflecting on the inadequacy of knowledge of drug dosages and the importance of completing drug courses. Common sites for obtaining drugs were the wards and OPDs of the hospital and the local pharmacies. $\sim 75 \%$ of the nursing staff would be confident of prescribing drugs based on their experience - as compared to the $\sim 15 \%$ of non-nursing staff who would. In conclusion, nursing staff are found to be self-medicating more and with higher confidence. There should be sensitization at time of recruitment of staff and conducting CMEs, hence making self-medication a more rational and effective tool of self-care.
\end{abstract}

Key-words: Antibiotic resistance, Self-medication, Support staff.

Key-message: This study was planned with the objective of evaluating self-medication practices in the support staff of a tertiary care hospital and
PICTORIAL ABSTRACT

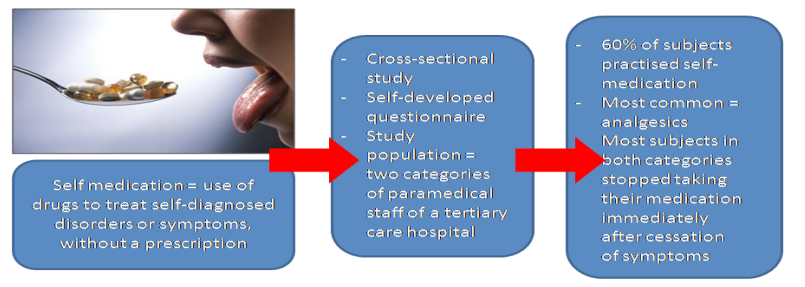

to study the prevalence of self-medication and knowledge of drug ADRs and antibiotic resistance among them.

Correspondence :

Gala Khushboo S

Department of Pharmacology, Grant Medical College, Mumbai 400013, Maharashtra India.

Mobile : 07506599104

E-mail: khushboogala18@gmail.com

DOI: 10.5530/jyp.2016.1.6

\section{INTRODUCTION}

The concept of self-treatment goes down to pre-historical times, where man had been using naturally available substances to rid himself of diseases. In the modern era of allopathy, self-medication with commonly available drugs is a widespread phenomenon. Self-medication is defined as the use of drugs to treat self-diagnosed disorders or symptoms, or the intermittent or continued use of a prescribed drug for chronic or recurrent disease or symptoms. ${ }^{1}$

Self-medication studies conducted amongst physicians and medical students have showed a high prevalence of self-medication amongst medical workers and could be regarded as an occupational hazard for the medical profession. Several studies have reported that inappropr ate self-medication results in wastage of resources and entails serious health hazards such as adverse drug reactions, prolonged suffering and drug dependence. ${ }^{1,2}$

On the other hand, if done appropriately, self-medication can readily relieve acute medical problems, can save the time spent in waiting to see a doctor, may be economical and can even save lives in acute conditions. ${ }^{1}$ This is especially important when it comes to medical field workers, who may be exposed to a larger amount of pathogens as compared to a lay man. The support staff of a tertiary care hospital, where drugs are freely available not only in the wards and OPDs, but also in the nearby chemist shops, has easy access to a large number of medications. Due to the na- ture of their work and the work environment, they also have a good idea about drug dosages, indications and contraindications. Being employed in an urban tertiary care hospital means that they are aware of the different brands of common generic drugs. Hence, self-medication amongst them has a different prevalence as compared to the general populace.

This study was planned with the objective of evaluating self-medication practices in the support staff of a tertiary care hospital and to study the prevalence of self-medication and knowledge of drug ADRs and antibiotic resistance amongst them.

\section{MATERIALS AND METHODS}

The study was carried out after approval from the Institutional Ethics Committee (Ethical Committee Letter No. IEC/Pharm/687/2013). This was a survey-based study in which a self-developed questionnaire consisting of both open-ended and close-ended items was used. Study population consisted of support staff from the medical wards of a tertiary care hospital in urban Mumbai, Maharashtra. Totally 100 were enrolled in the study and they were randomly divided in to two groups viz., category I (Nursing staff) and category II (Non nursing staff) Total sample size was 100 (each category $=50$ samples).

Subjects who are working in the hospital or in any other tertiary care hospital for more than a period of one year and comfortable with read- 
ing and understanding either English/Hindi were included in the study. Work experience in a tertiary care hospital for less than one year was excluded from the study. Subjects were explained the nature and purpose of the study. The procedure of completing the questionnaire was explained and informed consent was taken before starting the study. Subjects were given a choice between English and Hindi versions of the questionnaire, as per their comfort with the language.

\section{STATISTICAL METHODS}

Stata (version: 13.1) software as used for statistical analysis. Data summarised and Pearson's and Chi-Square test was used for $2 \times 2$ tables, as a test of significance. When the expected value in a cell is $<5$, Fisher's Exact test (tailed) was used as a test of significance and odds ratios and 95\% Confidence Intervals have been calculated. A P value of $<0.05$ is deemed statistically significant.

\section{RESULTS}

Percentage of participants practising self-medication was: Cat I - $\mathbf{6 8 \%}$ (34/50); Cat II - 52\% (26/50). The difference was not found to be statistically significant. $[p=0.102$; or $(95 \% C I)=1.96(0.81-4.80]$

The two categories were compared with respect to their self-medication practices in 5 drug groups, namely antibiotics, analgesics, anti histaminics, G.I. medication and nutritional supplements. (Graph 1)

It is observed that a higher number of subjects in Cat I were self-medicating with antibiotics (67.6\%) as compared to Cat II (19.2\%). The percentage of subjects self-medicating with all drug groups was higher in Cat I as compared to Cat II, except for G.I. medication, where Cat II (53.9\%) was higher than Cat I (38.2\%).

Subjects of Cat I show a significantly higher percentage in selecting drugs based on recommendations by co-working doctors and resident

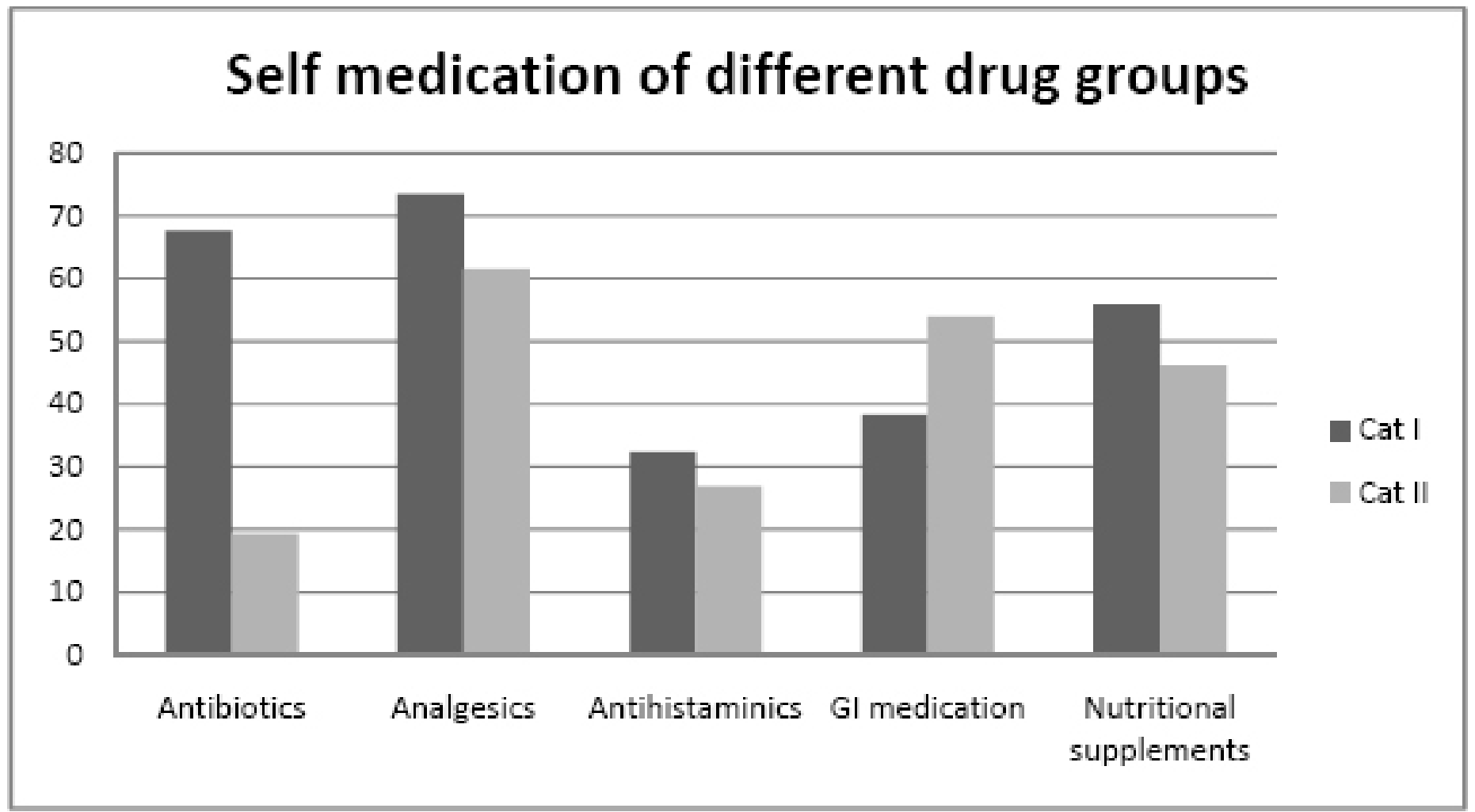

Graph 1: Percentage of subjects self-medicating with the different groups of drugs

Table 1: Different criteria for selection of drugs

\begin{tabular}{ccc}
\hline Criterion for selection & Cat I & Cat II \\
\hline $\begin{array}{c}\text { Recommendation by doctor/resident doctor } \\
{[p=0.007 ; \text { OR }(95 \% C I)=8.4(1.6-82.2)]}\end{array}$ & $38.84 \%$ & $4 \%$ \\
Recommendation by pharmacist & $23.48 \%$ & $6.25 \%$ \\
$\quad$ Opinion of colleagues/friends & $35.25 \%$ & $5.71 \%$ \\
{$[p=0.035 ;$ OR $(95 \%$ CI $)=4.18(0.93-25.61)]$} & & \\
Own experience & $38.09 \%$ & $26.89 \%$ \\
Previous doctor's prescription & $24.22 \%$ & $44.52 \%$ \\
The advertisement & $12.45 \%$ & $12.62 \%$ \\
\hline
\end{tabular}

Table 2: Cessation of drug intake $(*$ Statistically significant difference)

\begin{tabular}{ccc}
\hline Cessation of drug intake & Cat I & Cat II \\
\hline After a few days regardless of the outcome & $3.63 \%$ & $20.48 \%$ \\
After symptoms disappeared & $53.76 \%$ & $51.71 \%$ \\
A few days after the symptoms disappeared ${ }^{*}$ & $16.36 \%$ & $0 \%$ \\
$\quad[P=0.031)]$ & & \\
After drugs ran out & $26.21 \%$ & $12.08 \%$ \\
At the completion of the course & $16.71 \%$ & $18.58 \%$ \\
\hline
\end{tabular}


doctors $[p=0.007 ; \mathrm{OR}(95 \% C I)=8.4(1.6-82.2)]$, and opinions of colleagues/friends $[p=0.035 ; O R(95 \% C I)=4.18(0.93-25.61)] . \mathrm{A}$ similar percentage of subjects in both categories select drugs based on their own experience and advertisements. A higher percentage of Cat II subjects select drugs based on a previous doctor' prescription (44.5\%) as compared to Cat I (24.2\%). (Table 1)

It is seen that the knowledge of dosage in Cat I is by experience from administering doses to patients in wards/ OPDs is significantly higher than Cat II $[p=0.008]$, and by consulting a doctor/resident doctor $[p=0.008$; OR(95\% CI $)=7.42(1.39-73.07)]$.Cat II, on the other hand, has a significantly higher percentage of guessing of the dosage. $[p=0.0068 ;$ OR $(95 \% C I)=0.18(0.04-0.77)]$ (Table 2)

Nearly half of the subjects in both categories have stopped taking their medication after disappearance of symptoms (Cat I - 53.76\%, Cat II -
$51.71 \%$ ). Only $16.71 \%$ of Cat I and $18.58 \%$ of Cat II subjects stopped taking the drug after completing the course.

It is observed that a very highly significantly larger number of subjects from Cat I obtain drugs from the wards/OPDs as compared to Cat II $[p=0.0052$; OR $(95 \% C I)=5.06(1.37-19.73)]$.

A high and nearly equal number of subjects from both categories use drugs leftover from previous prescriptions ( $68 \%)$.

Graph 2 show awareness levels about ADRs and antibiotic resistance. Cat I shows higher awareness on both topics.

A similar number of both categories of subjects experienced ADRs while self-medicating (Cat I - 23.53\%, Cat II - 23.08\%). The methods of resolving ADRs are shown in Table 3.

When asked if the subject would continue to self-medicate if made aware about ADRs, $67.65 \%$ of Cat I and $26.92 \%$ of Cat II said they

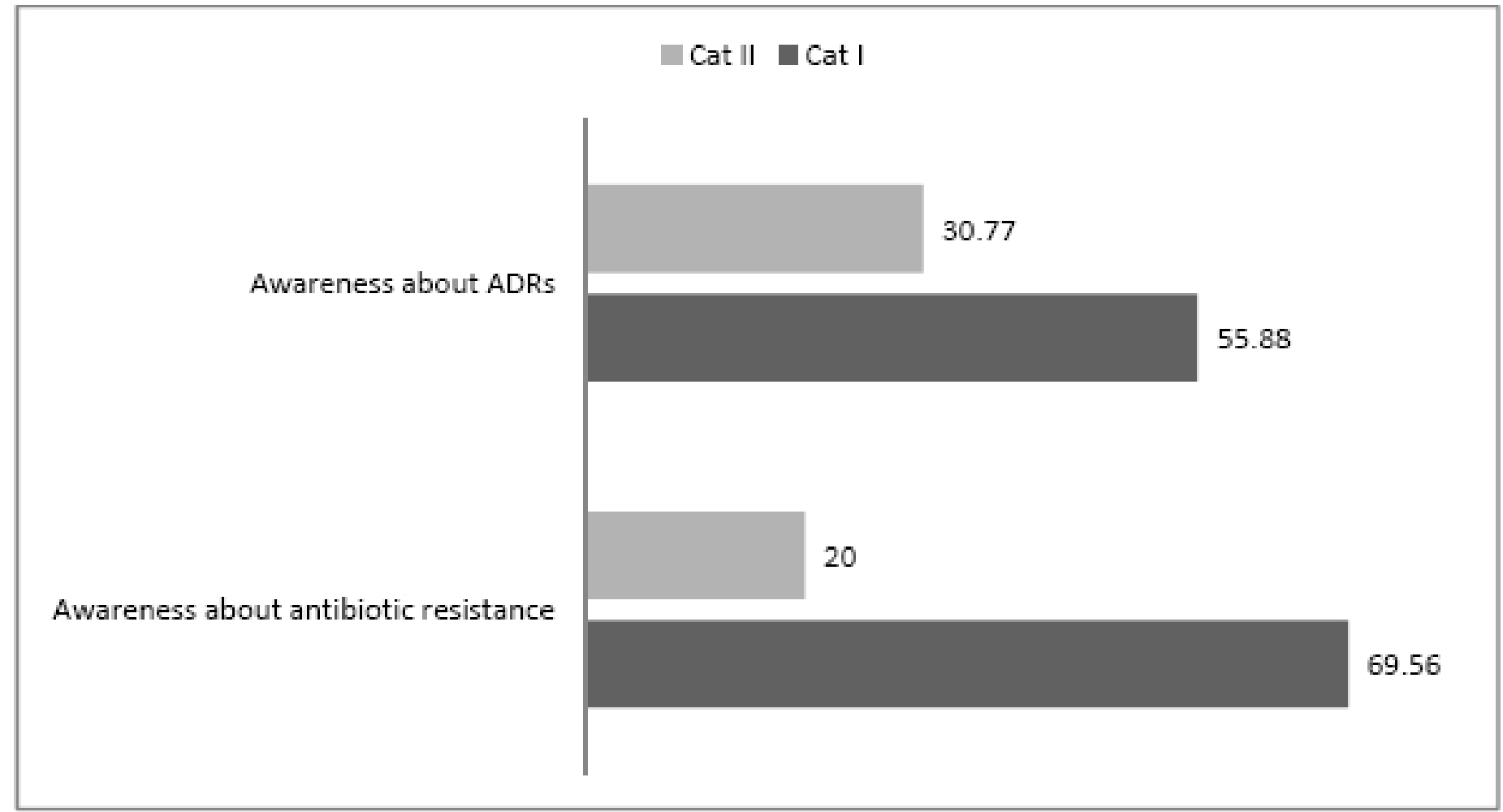

Graph 2: Awareness about ADRs and antibiotic resistance (expressed in percentages)

Table 3: Methods of resolving ADRs

\begin{tabular}{ccc}
\hline & Cat I & Cat II \\
\hline Stopping medication & $37.5 \%$ & $66 \%$ \\
Consulting the doctor & $62.5 \%$ & $37 \%$ \\
Self-medicating for the ADR in question & $0 \%$ & $0 \%$ \\
\hline
\end{tabular}

would. There was a highly significant difference here $[p=0.0018$; $O R(95 \%$ CI $)=5.68(1.63-20.67)]$.

The subjects were asked if they feel they can treat common infectious diseases by themselves. The responses are recorded in Table 9.1. It is interesting to note that $52.17 \%$ of Cat I subjects feel they can treat a disease by themselves, while $0 \%$ of Cat II feel they have the knowledge and aptitude to treat a disease.

When asked if the subjects would prescribe drugs based on their experience, $73.53 \%$ of Cat I and $15.38 \%$ of Cat II said they would.
This was a very highly significant difference. $[p>0.001 ; O R(95 \% C I)$ $=15.28(3.61-74.04)]$.

\section{DISCUSSION}

We see that a high percentage of the nursing staff is self-medicating with antibiotics $(\sim 68 \%)$. This finding is in accordance with other studies which also show that use of antibiotics is higher among medical personnel. ${ }^{5}$ Although the awareness levels about antibiotic resistance are high among the nursing staff ( $\sim 70 \%$ are aware about antibiotic resistance), such a widespread and possibly indiscriminate usage of antibiotics raises questions about the growing microbial resistance amongst the populace. The antibiotic usage by the non-nursing staff is lower ( 20\%), probably due to their ignorance of these higher drugs.

It is seen that in both categories of subjects, self-medication with analgesics is the most prevalent. This is in concordance with other studies on self-medication with different samples. ${ }^{2,4}$ It is interesting to note that the use of GI drugs is more in the non-nursing staff than the nursing staff. 
The commonest indication for use of these drugs in both categories of subjects is diarrhoea, which may be ascribed to poor eating and hygiene habits and rampant infection by pathogens.

Almost $50 \%$ of the subjects self-medicate with nutritional supplements, the most used drugs being vitamin supplements. Whether nutritional supplements are used for actual need or just as a placebo remains a question. As mentioned before, there is higher tendency to select the nutritional supplement by means of advertisements (Cat I $-57.89 \%$, Cat II $-41.67 \%)$ as compared to other drugs. The social impact of advertising of drugs may be worth addressing, in this regard.

When asked about how they know the dosage of the drug they are selfmedicating with, the non-nursing staff responded that they mainly guess the dosage. This is contrasting to the responses given by the nursing staff, which include by experience from administering medicines to patients, and by recommendations from doctors. A good number from both categories also rely $(\sim 35 \%)$ on their own past experience. This implies that self-medication of similar drugs is quite frequently and repeatedly done by both categories of subjects.

As mentioned previously, around half of the subjects in both categories stop take their medication immediately after cessation of symptoms. This reflects on the inadequacy of knowledge regarding the drug dosages and the importance of completing the course of the drug. This fact is especially important with regards to antibiotics, in which incomplete courses could result in development of resistance.

The commonest sites for obtaining drugs, as expected, are the wards and OPDs of the hospital and the local pharmacies. A high percentage of subjects also use drugs left over from previous prescriptions. This may imply noncompliance of patients with the drug schedules prescribed by the doctors.

Nearly $20 \%$ of both categories of subjects have faced ADRs while selfmedicating. It is interesting to note while majority of the nursing staff prefer consulting a doctor in case of the occurrence of an ADR, the nonnursing staff prefer stopping the medication. This reflects on the confidence of the subject while using a medication - which correlates well with the fact that nearly $70 \%$ of the nursing staff said that they would continue to self-medicate even if educated about ADRs (as opposed to only $27 \%$ of the non-nursing staff).

More than half of the nursing staff has responded that they think they can treat common infectious diseases by themselves, as compared to the $0 \%$ of the non-nursing staff who think they can. This again points to the confidence of the nursing staff while self-medicating. In concordance with this result is the fact that $\sim 75 \%$ of the nursing staff would be confident of prescribing drugs based on their experience - as compared to the $15 \%$ of non-nursing staff who would.

There definitely needs to be sensitization at time of recruitment of staff, to ensure that there is no misuse of freely available medication. Proper auditing of drugs, especially newer generation antibiotics, to curtail misuse should be followed in major hospitals. A definitive step could be hosting CMEs to increase awareness of the staff about antibiotic resistance and drug ADRs and at the same time enhance their knowledge about common drugs, hence making self-medication a more rational and effective tool of self-care.

\section{SUMMARY}

- There is a high rate of self medication in tertiary care hospitals, and most commonly used drugs for self medication were analgesics.

- Nursing staff had superior knowledge and awareness about drugs and their usage and greater confidence while self medicating.

- Most subjects stopped taking their medication immediately after cessation of symptoms, reflecting on the inadequacy of knowledge of drug dosages and the importance of completing drug courses.

- Common sites for obtaining drugs were the wards and OPDs of the hospital and the local pharmacies.

\section{ABBREVIATIONS USED}

OPD - Out Patient Department ; ADR - Adverse Drug Reaction ; CME - Continuing Medical Education.

\section{ABOUT AUTHOR}

Khushboo Gala is a MBBS student at Grant Medical College and Sir J. J. Group of hospitals, Mumbai. She is interested in clinical pharmacology and associated research.

\section{REFERENCES}

1. World Health Organization. Guidelines for the Regulatory Assessment of Medicinal Products for use in Self Medication.WHO/EDM/QSM/00.1

2. Hughes CM, McElnay JC, Fleming GF. Benefits and risks of self-medication. Drug Saf.2001; 24:1027-37.

3. Zafar SN, Syed R, Waqar S, Zubairi AJ, Vaqar T, Shaikh M, et al. Self-medication amongst University Students of Karachi: Prevalence, Knowledge and Attitudes. J Pak Med Assoc. 2008; 58(4): 214-7.

4. Vedrana AV, Vladimir T, Zdravko L. Content of home pharmacies and self medication practices in households of pharmacy and medical students in Zagreb, Croatia: findings in 2001 with a reference to 1977. Croat Med J. 2005; 46: 74-80.

5. Deshpande SG, Tiwari R. Self medication - A growing concern. Indian J Med Sci 1997; 51: 93-6.

6. Henry J, Handu SS, Khalid AJ, Khaja ASO, Sequeira RP. Evaluation of the Knowledge, Attitude and Practice of Self-Medication among First-Year Medical Students. Med PrincPract. 2006;15:270-5

7. Olayemi OJ, Olayinka BO, Musa Al. Evaluation of Antibiotic Self-Medication Pattern amongst Undergraduate Students of Ahmadu Bello University (Main Campus), Zaria. Res J Appl Sci Eng Tech. 2010; 2(1): 35-8.
8. Sawalha AF. A descriptive study of self-medication practices among Palestinian medical and nonmedical university students. Res Social Adm Pharm. 2008; 2:164-72.

9. Figueiras A, Caamano F, Gestal OJJ. Sociodemographic factors related to selfmedication in Spain. Eur J Epidemiol. 2000; 16(1):19-26.

10. Shankar PR, Partha P, Shenoy N. Self-medication and non-doctor prescription practices in Pokhara valley, Western Nepal: a questionnaire based study. BMC. Family Practice 2002;3:17.

11. Khantzian, EJ, Mack JF, \& Schatzberg AF. Heroin use as an attempt to cope: Clinical observations. Am J Psychiatry, 1974;131:160-4.

12. Khantzian, EJ. The self-medication hypothesis revisited: The dually diagnosed patient. Prim Psychiatry, 2003;10:47-48, 53-4.

13. Awad Abdelmoneim, Idris Eltayeb, Lloyd Matowe, Lukman Thalib. Self-medication with antibiotics and antimalarials in the community of Khartoum State, Sudan. J Pharm Pharm Sci. Year missing ;8(2): 326-31.

14. Nalini, G. K. Self-Medication among Allopathic medical Doctors in Karnataka India. BJMP. 2010; 3(2):325. 
15. Sontakke SD, Bajait CS, Pimpalkhute SA, Jaiswal KM, Jaiswal SR. Comparative study of evaluation of self-medication practices in first and third year medical students. Int J Biol Med Res. 2011; 2(2): 561-4.

16. Barros AR, Griep RH, Rotenberg L. Self-medication among nursing workers from public hospitals. Rev Lat Am Enfermagem. 2009;17(6):1015-22.
17. Gholap Manisha C, Mohite Vaishali R. Assess the self medication practices among staff nurses. Department of Medical Surgical Nursing, Krishna Institute of Medical Sciences, Deemed University, Krishna Institute of Nursing Sciences, Karad, India

18. Fox JM._Use of analgesics in self-medication. Therapie: 2002;57(2):115-8. 